\title{
Haploid biotechnology in the selection of Triticum aestivum $\mathrm{L}$.
}

Anapiyayev B.B. ${ }^{1}$, Iskakova K.M. ${ }^{2}$, Beysenbek E.B. ${ }^{1}$, Akhmetova A.B. ${ }^{2}$

${ }^{1}$ Satbayev University, Almaty, Kazakhstan

${ }^{2}$ Kazakh National Agrarian University, Almaty, Kazakhstan

*e-mail:bak_anapiyayev@mail.ru

Haploids are unique objects for cell selection and genetic engineering. Our work presents the results of the use of haploid biotechnology in the practical selection of Triticum aestivum L. for resistance to rust diseases. In the first series of experiments, the field resistance of the original wheat varieties and hybrids under conditions of an infectious nursery was determined. The greatest resistance to rust was shown by the isogenic line Lr 24: to yellow rust $-2 / 20$, to brown $-2 / 10$, and was immune to stem rust. Similar values of resistance to yellow and brown rust showed isogenic lines Lr 19, Lr 25. By resistance to stem rust, the isogenic line $\operatorname{Lr} 25$ was weakly susceptible $-2 / 20$, and $\operatorname{Lr} 19$ showed an average susceptibility - 3/30, respectively. In the second series of experiments, the varieties of common wheat were crossed with donors of effective resistance genes - isogenic lines Lr 19, Lr 24. Valuable wheat hybrids were genetically stabilized by haploid biotechnology based on in vitro culture of isolated microspores. As a result of studies on the use of haploid biotechnology based on in vitro culture of isolated microspores in the selection of wheat Triticum aestivum L. for resistance to rust diseases, we obtained embryoids, morphogenic calli and regenerant plants from which $\mathrm{DH}$ lines were created. To determine the resistance of $\mathrm{DH}$ lines to rust diseases, they were studied in the conditions of an infectious nursery of South-East Kazakhstan. As a result of the studies conducted in an infectious nursery, DH wheat lines were selected, which showed a high level of resistance to rust diseases. Thus, it was shown that haploid biotechnology is an effective method for accelerating the selection process and rapid genetic stabilization of promising hybrids. In the early stages of the selection process, wheat DH lines carrying the genes for resistance to rust diseases were selected. 